\title{
Research Article Symmetry Classification of First Integrals for Scalar Linearizable Second-Order ODEs
}

\author{
K. S. Mahomed and E. Momoniat \\ Centre for Differential Equations, Continuum Mechanics and Applications, \\ School of Computational and Applied Mathematics, University of the Witwatersrand, Private Bag 3, \\ Wits 2050, Johannesburg, South Africa \\ Correspondence should be addressed to K. S. Mahomed, komalmajeed@hotmail.com
}

Received 2 September 2012; Accepted 1 October 2012

Academic Editor: Fazal M. Mahomed

Copyright (C) 2012 K. S. Mahomed and E. Momoniat. This is an open access article distributed under the Creative Commons Attribution License, which permits unrestricted use, distribution, and reproduction in any medium, provided the original work is properly cited.

\begin{abstract}
Symmetries of the fundamental first integrals for scalar second-order ordinary differential equations (ODEs) which are linear or linearizable by point transformations have already been obtained. Firstly we show how one can determine the relationship between the symmetries and the first integrals of linear or linearizable scalar ODEs of order two. Secondly, a complete classification of point symmetries of first integrals of such linear ODEs is studied. As a consequence, we provide a counting theorem for the point symmetries of first integrals of scalar linearizable second-order ODEs. We show that there exists the $0-, 1-, 2-$, or 3-point symmetry cases. It is shown that the maximal algebra case is unique.
\end{abstract}

\section{Introduction}

First integrals or constants of the motion of ordinary differential equations (ODEs) are quite an active and interesting area of research at the present time. Whenever one is dealing with differential equations and especially with their solutions, you have to deal with first integrals. In fact, first integrals are the first primary steps towards finding the solutions of differential equations. First integrals have great importance in mechanics as it deals with second-order systems of equations and constants of the motion. The maximum number of symmetries for scalar $n$ th-order ODEs was investigated by Lie [1] (see Mahomed [2]). Lie showed that scalar first-order ODEs have infinite number of point symmetries. In the case of scalar second-order ODEs Lie proved that the maximum is eight, and this is achieved by the free particle and indeed linearizable by point transformation equations. In recent work by Mahomed and Leach [3], they discovered the symmetries of the maximal cases of scalar linear $n$ th-order ODEs, $n \geq 3$. These cases are $n+1, n+2$, and $n+4$. There is yet another 
contribution by Leach and Mahomed [4], in which they have found that the Lie algebra of the fundamental first integrals and their quotient of scalar linear second-order ODEs are three-dimensional and have very interesting properties. This also applies to linearizable by invertible transformations second-order ODEs which are given as examples in their paper. In the case of scalar third-order linear ODEs, Govinder and Leach [5] provided the algebraic structure of the basic first integrals. They showed that in the three equivalence classes each has certain first integrals with a specific number of point symmetries. So far, none of these authors consider the classification of the symmetries of first integrals of scalar linear $n$ thorder ODEs, $n \geq 1$, nor even investigate what could be the maximal numbers of symmetries for the first integrals of these linear or linearizable equations. They do give insights into the algebraic structure of the fundamental first integrals and in some cases their quotients.

In this paper we give the complete classification of point symmetries for the first integrals of scalar linear second-order ODEs and the relationship between the symmetries and first integrals. For this purpose we use the projective transformations to find the different cases of symmetries for the first integrals of scalar second-order ODEs which are linear or linearizable by point transformations. Since all scalar second-order ODEs which are linear or linearizable by point transformations are transformable to the free particle equation, we utilize this as our base ODE. We find that there are the no-symmetry, one-symmetry, twosymmetry, and unique three-symmetry cases.

It is well known that the second-order ODE

$$
E\left(x, y, y^{\prime}, y^{\prime \prime}\right)=0
$$

is invariant under the infinitesimal generator

$$
X=\xi(x, y) \frac{\partial}{\partial x}+\eta(x, y) \frac{\partial}{\partial y}
$$

if and only if

$$
\left.X^{[2]} E\right|_{E=0}=0
$$

where

$$
X^{[2]}=X+\zeta_{1} \frac{\partial}{\partial y^{\prime}}+\zeta_{2} \frac{\partial}{\partial y^{\prime \prime}}
$$

with

$$
\begin{aligned}
& \zeta_{1}=D_{x}(\eta)-y^{\prime} D_{x}(\xi), \\
& \zeta_{2}=D_{x}\left(\zeta_{1}\right)-y^{\prime \prime} D_{x}(\xi)
\end{aligned}
$$

in which $D_{x}$ is the total differentiation operator, is called the second prolongation of the generator $X$. 
Now we can say that (1.2) is the point symmetry of (1.1), whereas, in the case of first integrals, the first integral

$$
I=f\left(x, y, y^{\prime}\right)
$$

of the ODE (1.1), is annihilated by $X$, that is, (1.2) is the symmetry generator of (1.6) if and only if

$$
X^{[1]} I=0 .
$$

Here $X$ annihilates $I$ and does not leave it invariant as in the case of symmetries of equations. Note that the procedure for finding symmetries of ODEs is different to that of finding symmetries of first integrals. In fact the symmetries of the first integrals are a subalgebra of the symmetries of the equation itself (see Kara and Mahomed [6]).

It is essential to point out that (1.1) is linearizable by point transformation to the free particle equation if and only if it is cubic in the first derivatives as

$$
y^{\prime \prime}=A(x, y) y^{\prime 3}+B(x, y) y^{\prime 2}+C(x, y) y^{\prime}+D(x, y)
$$

where the functions from $A$ to $D$ satisfy the invariant conditions (see Tresse [7] and also [8])

$$
\begin{aligned}
& 3 A_{x x}+3 A_{x} C-3 A_{y} D+3 A C_{x}+C_{y y}-6 A D_{y}+B C_{y}-2 B B_{x}-2 B_{x y}=0, \\
& 6 A_{x} D-3 B_{y} D+3 A D_{x}+B_{x x}-2 C_{x y}-3 B D_{y}+3 D_{y y}+2 C C_{y}-C B_{x}=0 .
\end{aligned}
$$

As an example we revisit the well-known modified Emden equation which has eight point symmetries [9]

$$
y^{\prime \prime}+3 y y^{\prime}+y^{3}=0
$$

This ODE satisfies (1.8) and (1.9) and is reducible to the free particle equation $\bar{y}^{\prime \prime}=0$ via the map (see [9])

$$
\bar{x}=x-\frac{1}{y}, \quad \bar{y}=\frac{1}{2} x^{2}-\frac{x}{y}
$$

Therefore in the sequel we consider the free particle equation as representative of all linearizable by point transformations scalar second-order ODEs.

In the next section we give the classifying relation for the symmetries of the first integrals of the free particle equation. 


\section{Symmetries of the Fundamental First Integrals}

We consider the free particle equation

$$
y^{\prime \prime}=0
$$

which has the maximum number of symmetries, namely, eight given by (we list them here as we use these in what follows)

$$
\begin{gathered}
X_{1}=\frac{\partial}{\partial x} \\
X_{2}=\frac{\partial}{\partial y} \\
X_{3}=x \frac{\partial}{\partial x} \\
X_{4}=y \frac{\partial}{\partial y} \\
X_{5}=x \frac{\partial}{\partial y} \\
X_{6}=y \frac{\partial}{\partial x} \\
X_{7}=x^{2} \frac{\partial}{\partial x}+x y \frac{\partial}{\partial y} \\
X_{8}=x y \frac{\partial}{\partial x}+y^{2} \frac{\partial}{\partial y} .
\end{gathered}
$$

It is clear that the free particle equation (2.1) has two functionally independent first integrals

$$
\begin{gathered}
I_{1}=y^{\prime}, \\
I_{2}=x y^{\prime}-y .
\end{gathered}
$$

The first integral (2.3a) has three symmetries [4]

$$
\begin{gathered}
X_{1}=\frac{\partial}{\partial x} \\
X_{2}=\frac{\partial}{\partial y} \\
X_{3}=x \frac{\partial}{\partial x}+y \frac{\partial}{\partial y}
\end{gathered}
$$


and (2.3b) also has three symmetries [4]

$$
\begin{gathered}
G_{1}=x \frac{\partial}{\partial x} \\
G_{2}=x \frac{\partial}{\partial y} \\
G_{3}=x^{2} \frac{\partial}{\partial x}+x y \frac{\partial}{\partial y} .
\end{gathered}
$$

We observe that the symmetries of the first integral of (2.3a) are the same as that of (2.3b) if we multiply the symmetries of (2.3a) by $x$ which is the multiplier or characteristic of the free particle equation that results in the integral $I_{2}$.

Let us see what happens if we find the symmetries of the quotient of the first integrals ((2.3a) and (2.3b)), namely,

$$
\frac{I_{2}}{I_{1}}=x-\frac{y}{y^{\prime}} .
$$

As shown in [4], (2.6) possesses three symmetries as well. These are

$$
\begin{gathered}
\Upsilon_{1}=y \frac{\partial}{\partial x}, \\
\Upsilon_{2}=y \frac{\partial}{\partial y^{\prime}} \\
\Upsilon_{3}=x y \frac{\partial}{\partial x}+y^{2} \frac{\partial}{\partial y},
\end{gathered}
$$

which are the same as the symmetries (2.4) if we multiply the symmetries of (2.3a) by $y$. However, this is not a multiplier of our equation.

It was demonstrated, in the seminal paper [4], that the Lie algebras of the symmetries of the first integrals $I_{1}, I_{2}$ and their quotient $I_{2} / I_{1}$ are isomorphic. Also each triplet (2.4), (2.5), and (2.7) can be mapped into the other by a projective transformation. Furthermore, it was noted in [4] that the three triplets together generate the Lie algebra $\operatorname{sl}(3, R)$ of the free particle equation.

\section{Classifying Relation for the Symmetries}

We know (see [4]) the symmetries of the functionally independent first integrals $I_{1}$ and $I_{2}$ or their quotient of the free particle equations. These are what we briefly reviewed and commented on in the previous section. Now the question arises if we want to know the symmetry properties of say the product $I_{1} I_{2}$. We then need to compute them from first principles by using the symmetry condition. Instead of doing this each time from the beginning principles, can one obtain the relationship between the symmetries and first integrals? This is what we do here. The benefit of having such a relation enables us to also classify the first integrals of the free particle equation in terms of their point symmetries. 
Let then $F$ be an arbitrary function of $I_{1}$ and $I_{2}$, namely, $F=F\left(I_{1}, I_{2}\right)$. The symmetry of this general function of the first integrals is

$$
X^{[1]} F=X^{[1]} I_{1} \frac{\partial F}{\partial I_{1}}+X^{[1]} I_{2} \frac{\partial F}{\partial I_{2}}=0
$$

where

$$
\begin{gathered}
X^{[1]} I_{1}=\left[\xi \frac{\partial}{\partial x}+\eta \frac{\partial}{\partial y}+\zeta_{x} \frac{\partial}{\partial y^{\prime}}\right] y^{\prime}=\zeta_{x} \\
X^{[1]} I_{2}=\left[\xi \frac{\partial}{\partial x}+\eta \frac{\partial}{\partial y}+\zeta_{x} \frac{\partial}{\partial y^{\prime}}\right]\left(x y^{\prime}-y\right)=\xi y^{\prime}+x \zeta_{x}-\eta .
\end{gathered}
$$

Now $\xi, \eta$, and $\zeta_{x}$ are

$$
\begin{gathered}
\xi=a_{1}+x a_{3}+y a_{6}+x^{2} a_{7}+x y a_{8} \\
\eta=a_{2}+y a_{4}+x a_{5}+x y a_{7}+y^{2} a_{8} \\
\zeta_{x}=-y^{\prime} a_{3}+y^{\prime} a_{4}+a_{5}-y^{\prime 2} a_{6}+\left(y-x y^{\prime}\right) a_{7}+\left(y y^{\prime}-x y^{\prime 2}\right) a_{8} .
\end{gathered}
$$

These are the coefficients of $X^{[1]}$ which are obtained by setting

$$
X^{[1]}=\sum_{i=1}^{8} a_{i} X_{i}^{[1]}
$$

where $X_{i}$ s are the free particle symmetries as given in (2.2), and the $a_{i}$ s are constants. The reason for this is that the symmetries of the first integrals are always the symmetries of the free particle equation (see [6]).

After substituting the values of $X^{[1]} I_{1}, X^{[1]} I_{2}$ as in (3.2), with $\xi, \eta$, and $\zeta_{x}$ as in (3.3), in (3.1), we get after some calculations

$$
\begin{aligned}
& {\left[-y^{\prime} a_{3}+y^{\prime} a_{4}+a_{5}-y^{\prime 2} a_{6}+\left(y-x y^{\prime}\right) a_{7}+\left(y y^{\prime}-x y^{\prime 2}\right) a_{8}\right] \frac{\partial F}{\partial I_{1}}} \\
& +\left[\left(a_{1}+x a_{3}+y a_{6}+x^{2} a_{7}+x y a_{8}\right) y^{\prime}\right. \\
& +\left(-y^{\prime} a_{3}+y^{\prime} a_{4}+a_{5}-y^{\prime 2} a_{6}+\left(y-x y^{\prime}\right) a_{7}+\left(y y^{\prime}-x y^{\prime 2}\right) a_{8}\right) x \\
& \left.\quad-\left(a_{2}+y a_{4}+x a_{5}+x y a_{7}+y^{2} a_{8}\right)\right] \frac{\partial F}{\partial I_{2}}=0 .
\end{aligned}
$$


Then by using the relations $I_{1}=y^{\prime}$ and $I_{2}=x y^{\prime}-y$ from $((2.3 \mathrm{a})$ and $(2.3 \mathrm{~b}))$, we finally arrive at the classifying relation

$$
\begin{aligned}
& \left(-I_{1} a_{3}+I_{1} a_{4}+a_{5}-I_{1}^{2} a_{6}-I_{2} a_{7}-I_{1} I_{2} a_{8}\right) \frac{\partial F}{\partial I_{1}} \\
& +\left(I_{1} a_{1}-a_{2}+I_{2} a_{4}-I_{1} I_{2} a_{6}-I_{2}^{2} a_{8}\right) \frac{\partial F}{\partial I_{2}}=0 .
\end{aligned}
$$

The relation (3.6) provides the relationship between the symmetries and first integrals of the free particle equation. We remind the reader that any symmetry of a first integral of the free particle equation is contained in the condition (3.6). We use this to classify the first integrals according to their symmetries.

\section{Symmetry Structure of First Integrals}

We invoke the classifying relation (3.6) to establish the number of symmetries possessed by the first integrals of the free particle equation.

There arise four cases.

Case 1 (no symmetry). If $F$ is any arbitrary function of $I_{1}$ and $I_{2}$, then $F_{I_{1}}$ and $F_{I_{2}}$ are not related to each other. In this case we have from (3.6) that

$$
\begin{gathered}
-I_{1} a_{3}+I_{1} a_{4}+a_{5}-I_{1}^{2} a_{6}-I_{2} a_{7}-I_{1} I_{2} a_{8}=0, \\
I_{1} a_{1}-a_{2}+I_{2} a_{4}-I_{1} I_{2} a_{6}-I_{2}^{2} a_{8}=0 .
\end{gathered}
$$

It is easy to see from (4.1) that all the as are zero. Therefore there exists no symmetry for this case.

As an illustrative example, if we take $F=I_{1} \ln I_{2}$, then (3.6) yields

$$
\begin{gathered}
\left(-I_{1} a_{3}+I_{1} a_{4}+a_{5}-I_{1}^{2} a_{6}-I_{2} a_{7}-I_{1} I_{2} a_{8}\right) I_{2} \ln I_{2} \\
+\left(I_{1} a_{1}-a_{2}+I_{2} a_{4}-I_{1} I_{2} a_{6}-I_{2}^{2} a_{8}\right) I_{1}=0 .
\end{gathered}
$$

This straightforwardly results in all the as being zero.

The results here are quite unexpected and surprising. One will not have imagined a zero symmetry case for a first integral of the simplest equation! The consequence of this is as follows.

If we set the first integral to be a constant as in the example, we have

$$
y^{\prime} \ln \left(x y^{\prime}-y\right)=C
$$

To integrate this kind of messy integral (4.3) and find the solution of the free particle equation from it is not easy. But this difficulty is avoidable. One does not usually obtain complicated first integral such as (4.3) in ones computation in the first place by using the approaches such as the direct method, Noether's theorem, and multiplier approach. 
Case 2 (one Symmetry). Firstly we notice that if $F$ satisfies the classifying relation (3.6), then $X$, which is a linear combination of the free particle generators, is a symmetry of this classifying relation. We also observe from (3.6) that if one has any of the free symmetry generators $X_{i}$ as a symmetry of a first integral of the equation, then one ends up with three symmetries! That is one can have more than one symmetry.

Say if we take $a_{2}$ arbitrary, that is, $X=\partial / \partial y$, then (3.6) yields (since $\partial F / \partial I_{2}=0$ and $\left.\partial F / \partial I_{1} \neq 0\right)$

$$
-I_{1} a_{3}+I_{1} a_{4}+a_{5}-I_{1}^{2} a_{6}-I_{2} a_{7}-I_{1} I_{2} a_{8}=0,
$$

which in turn implies that $a_{1}$ is arbitrary and $a_{3}=a_{4}$ as well. Thus we get more than one symmetry.

We in fact arrive at the three symmetries given in (2.4). The same applies for the other symmetries taken one at a time.

However, we do have several cases when exactly one symmetry occurs.

If we take $F=I_{1} I_{2}$ or any function of the product, then the relation (3.6) gives rise to exactly the symmetry

$$
X=2 x \frac{\partial}{\partial x}+y \frac{\partial}{\partial y}
$$

If we let $F=\exp \left(I_{1}^{2} I_{2}\right)$, then (3.6) results in only

$$
X=3 x \frac{\partial}{\partial x}+2 y \frac{\partial}{\partial y}
$$

As another simple example, if we set $F=I_{1} \exp \left(-I_{2}\right)$, then (3.6) implies the one symmetry

$$
X=x \frac{\partial}{\partial x}+\frac{\partial}{\partial y} .
$$

As a matter of interest there are infinitely many one-symmetry cases.

To see this we consider the first integral

$$
F=\frac{1}{2} I_{1}^{2}-a I_{2}, \quad a \neq 0 .
$$

The relation (3.6) then yields

$$
\begin{aligned}
& \left(-I_{1} a_{3}+I_{1} a_{4}+a_{5}-I_{1}^{2} a_{6}-I_{2} a_{7}-I_{1} I_{2} a_{8}\right) I_{1} \\
& \quad+\left(I_{1} a_{1}-a_{2}+I_{2} a_{4}-I_{1} I_{2} a_{6}-I_{2}^{2} a_{8}\right)(-a)=0 .
\end{aligned}
$$


Separation with respect to powers of $I_{1}$ and $I_{2}$ gives rise to $a a_{1}=a_{5}$. Therefore we have the parameter-dependent symmetry

$$
X=X_{1}+a X_{5}
$$

Yet a more complicated one is

$$
X=X_{1}+a X_{5}+a X_{6}, \quad a \neq 0 .
$$

This symmetry is associated with the first integral

$$
F=\frac{\left(I_{2} a-1\right)^{2}}{I_{1}^{2} a-a}
$$

which can be constructed just as before. Similarly, there are many possibilities for one symmetry.

Therefore the one symmetry case is not unique. Next we discuss the two symmetry case.

Case 3 (two symmetries). We have already seen from Case 2 that the translations in $x$ and $y$ symmetries further imply the uniform scaling symmetry. Thus one cannot have two symmetries of translations alone that are associated with a first integral of the free particle equation. Likewise the same applies for the translations in $y$ and the uniform scaling symmetries.

Further if we have the symmetries

$$
X=\frac{\partial}{\partial y}, \quad Y=x \frac{\partial}{\partial y}
$$

which forms the two-dimensional Abelian algebra, then $a_{2}$ and $a_{5}$ are arbitrary in (3.6). This directly gives

$$
\frac{\partial F}{\partial I_{1}}=\frac{\partial F}{\partial I_{2}}=0
$$

and hence no integral. This means that one does not have these types of symmetries admitted by any first integral of the free particle equation.

The same argument applies if we consider

$$
X=\frac{\partial}{\partial y}, \quad Y=y \frac{\partial}{\partial y}
$$

which forms a two-dimensional non-Abelian algebra. Here again this two-dimensional algebra is not admitted by any integral of the equation. 
So when do two symmetries occur for a first integral of the free particle equation? From the above it is clear that the simple type of symmetry combinations does not form two symmetries of an integral. Thus there have to be combinations of the symmetries. One such combination is

$$
\begin{aligned}
& X=\frac{\partial}{\partial x}-x \frac{\partial}{\partial x} \\
& Y=\frac{\partial}{\partial y}-x \frac{\partial}{\partial y}
\end{aligned}
$$

The Lie algebra formed by (4.16) is two dimensional with commutator $[X, Y]=-Y$. Here the combination of symmetries means that $a_{3}=-a_{1}$ and $a_{5}=-a_{2}$. The use of these in the relation (3.6) forces $F$ to satisfy the one condition

$$
\frac{\partial F}{\partial I_{1}}+\frac{\partial F}{\partial I_{2}}=0
$$

which gives the independent integral

$$
F=I_{2}-I_{1}
$$

Hence this $F$ admits two symmetries.

We now look at a case in which at least one of the symmetries has a parameter in it. This is provided by the operators

$$
\begin{gathered}
X=X_{1}+a X_{6}, \quad a \neq 0 \\
Y=X_{2}+a X_{4} .
\end{gathered}
$$

The symmetries (4.19) span a two-dimensional algebra with

$$
[X, Y]=-a X
$$

Here $F$ is given by

$$
F=a \frac{I_{2}}{I_{1}}-\frac{1}{I_{1}}
$$

We conclude by saying that the two symmetry case is not unique.

Case 4 (three symmetry). We now present a detailed study of possible three-dimensional algebra of symmetries admitted by first integrals of the free particle equation. Two essential deductions come out of our analysis. Firstly we show that the three-dimensional algebra admitted by a first integral is unique. Secondly we prove that three is the maximal dimension admitted by any integral.

We utilize the realizations of three-dimensional Lie algebras in the real plane given by Mahomed and Leach [10]. However, we use the notation given in Ibragimov and Mahomed 
Table 1: Realizations of three-dimensional algebras in the real plane.

\begin{tabular}{ll}
\hline & $p=\partial / \partial x$ and $q=\partial / \partial y$ \\
Algebra & Realizations in $(x, y)$ plane \\
\hline$L_{3 ; 1}$ & $X_{1}=q, X_{2}=x q, X_{3}=h(x) q$ \\
$L_{3 ; 2}$ & $X_{1}=q, X_{2}=p, X_{3}=x q$ \\
$L_{3 ; 3}^{I}$ & $X_{1}=q, X_{2}=p, X_{3}=x p+(x+y) q$ \\
$L_{3 ; 3}^{\mathrm{II}}$ & $X_{1}=q, X_{2}=x q, X_{3}=p+y q$ \\
$L_{3 ; 4}^{\mathrm{I}}$ & $X_{1}=p, X_{2}=q, X_{3}=x p$ \\
$L_{3 ; 4}^{\mathrm{II}}$ & $X_{1}=q, X_{2}=x q, X_{3}=x p+y q$ \\
$L_{3 ; 5}^{\mathrm{I}}$ & $X_{1}=p, X_{2}=q, X_{3}=x p+y q$ \\
$L_{3 ; 5}^{\mathrm{II}}$ & $X_{1}=q, X_{2}=x q, X_{3}=y q$ \\
$L_{3 ; 6}^{\mathrm{I}}$ & $X_{1}=p, X_{2}=q, X_{3}=x p+a y q, a \neq 0,1$ \\
$L_{3 ; 6}^{\mathrm{II}}$ & $X_{1}=q, X_{2}=x q, X_{3}=(1-a) x p+y q, a \neq 0,1$ \\
$L_{3 ; 7}^{\mathrm{I}}$ & $X_{1}=p, X_{2}=q, X_{3}=(b x+y) p+(b y-x) q$ \\
$L_{3 ; 7}^{\mathrm{II}}$ & $X_{1}=x q, X_{2}=q, X_{3}=\left(1+x^{2}\right) p+(x+b) y q$ \\
$L_{3 ; 8}^{\mathrm{I}}$ & $X_{1}=q, X_{2}=x p+y q, X_{3}=2 x y p+y^{2} q$ \\
$L_{3 ; 8}^{\mathrm{II}} q$ & $X_{1}=q, X_{2}=x p+y q, X_{3}=2 x y p+\left(y^{2}-x^{2}\right) q$ \\
$L_{3 ; 8}^{\mathrm{II}}$ & $X_{1}=q, X_{2}=x p+y q, X_{3}=2 x y p+\left(y^{2}+x^{2}\right) q$ \\
$L_{3 ; 8}^{\mathrm{IV}}$ & $X_{1}=q, X_{2}=y q, X_{3}=y^{2} q$ \\
$L_{3 ; 9}$ & $X_{1}=\left(1+x^{2}\right) p+x y q, X_{2}=x y p+\left(1+y^{2}\right) q$, \\
& $X_{3}=y p-x q$ \\
\hline
\end{tabular}

[11] (see also Mahomed [2]), that is, $L_{3 ; i}^{\alpha}$, where 3 refers to the dimension of the algebra, $i$ to the number of the algebra in some given ordering and $\alpha$ is the realization as an algebra may have more than one realization. For example, $L_{3 ; 4}^{\text {II }}$ denotes the second realization of the fourth Lie algebra of dimension 3.

All canonical forms of three-dimensional real Lie algebras in the plane is given in Table 1. This is taken from [2].

Remark 4.1. We point out that the Lie algebras $L_{3 ; 1}$ and $L_{3 ; 8}^{\mathrm{IV}}$ are not admitted by any scalar second-order ODE. Hence we do not consider these hereafter (see [10]).

Instead of using the realizations $L_{3 ; 8}^{\mathrm{I}}, L_{3 ; 8}^{\mathrm{II}}$, and $L_{3 ; 8}^{\mathrm{III}}$ given in Table 1, we use the free particle generators (see [12])

$$
\begin{aligned}
& X_{1}=p, \quad X_{2}=x p+\frac{1}{2} y q, \quad X_{3}=x^{2} p+x y q, \\
& X_{1}=p+x q, \quad X_{2}=x p+2 y q, \quad X_{3}=2\left(x^{2}-y\right) p+2 x y q, \\
& X_{1}=p-x q, \quad X_{2}=-x p+2 y q, \quad X_{3}=2\left(x^{2}+y\right) p+2 x y q \text {. }
\end{aligned}
$$

Therefore the realizations of three-dimensional algebras given in Table 1 by replacement of $L_{3 ; 8^{\prime}}^{\mathrm{I}} L_{3 ; 8}^{\mathrm{II}}$, and $L_{3 ; 8}^{\mathrm{III}}$ by their free particle operators (4.22), (4.23), and (4.24) above, except $L_{3 ; 1}$ and $L_{3 ; 8}^{\mathrm{IV}}$, are free particle symmetry generators. We utilize these in our analysis below. 
As $L_{3 ; 1}$ is not admitted by the free particle equation, we begin with $L_{3 ; 2}$. We want this algebra to be admitted by a first integral of the free particle equation. We utilize the classifying relation (3.6). Therefore $a_{1}, a_{2}$, and $a_{5}$ are arbitrary which imply that $F$ is constant. Hence this algebra is not admitted by any first integral.

The same applies to the algebras $L_{3 ; 3}^{\mathrm{I}}, L_{3 ; 3}^{\mathrm{II}}, L_{3 ; 4^{\prime}}^{\mathrm{I}} L_{3 ; 4^{\prime}}^{\mathrm{II}} L_{3 ; 5}^{\mathrm{II}}, L_{3 ; 6^{\prime}}^{\mathrm{I}} L_{3 ; 6^{\prime}}^{\mathrm{II}} L_{3 ; 7}^{\mathrm{I}}, L_{3 ; 7}^{\mathrm{II}}$ and $L_{3 ; 8^{\circ}}^{\mathrm{I}}$.

We separately consider the algebra $L_{3 ; 8}^{\mathrm{II}}$. We show that this algebra is not admitted by an integral as well. For what follows we utilize the free particle representation (4.23). We find that these operators correspond to $a_{1}=a_{5}, 2 a_{3}=a_{4}$, and $a_{6}=-a_{7}$. The substitution of the latter in the relation (3.6) results in the three conditions on $F$, namely,

$$
\begin{gathered}
I_{1} \frac{\partial F}{\partial I_{1}}+2 I_{2} \frac{\partial F}{\partial I_{2}}=0, \\
\frac{\partial F}{\partial I_{1}}+I_{1} \frac{\partial F}{\partial I_{2}}=0 \\
\left(I_{1}^{2}-I_{2}\right) \frac{\partial F}{\partial I_{1}}+I_{1} I_{2} \frac{\partial F}{\partial I_{2}}=0 .
\end{gathered}
$$

The first two imply that $F$ is constant which satisfy the third. Thus there is no algebra of this type admitted by a first integral of the free particle equation. The analysis for $L_{3 ; 8}^{\text {III }}$ is similar and this algebra too is not admitted.

For $L_{3 ; 9}$, the operators imply that $a_{6}=-a_{5}, a_{7}=a_{1}$, and $a_{8}=a_{2}$, the insertion of which into the relation (3.6) gives a condition on $F$ with $a_{1}, a_{2}$, and $a_{5}$ arbitrary. Then the result that $F$ must be constant arises. Thus this algebra is not admitted as well.

In the case of $L_{3 ; 5}^{\mathrm{I}}$ we have that $I_{1}$ has this algebra. This is precisely the algebra of the symmetries given in (2.4). We mention that the symmetries given in (2.5) and (2.7) also form the algebra $L_{3 ; 5}^{\mathrm{I}}$ as a projective transformation (see [4]) maps each of the representations to the one given in (2.4).

In conclusion, we have that the only three-dimensional algebra admitted by a first integral of the free particle equation is $L_{3 ; 5}^{\mathrm{I}}$.

We can state the following theorems.

Theorem 4.2. A first integral of the free particle or any scalar linearizable, by point transformation, second-order ODE admits a three-dimensional algebra if and only if the algebra is $L_{3 ; 5}^{I}$.

The proof follows from the preceding discussion. Also we note that this algebra $L_{3 ; 5}^{\mathrm{I}}$ is admitted by the integrals $I_{1}, I_{2}$ or $I_{2} / I_{1}$.

Theorem 4.3. The maximum dimension of the algebra admitted by any first integral of the free particle or any scalar linearizable, by point transformation, second-order ODE is three, and the algebra is $L_{3 ; 5}^{I}$.

Proof. A first integral of the free particle or scalar second-order ODE, which is linearizable by point transformation, cannot admit a maximal algebra of dimension more than three since the functionally independent integrals or their quotient has the unique three-dimensional algebra which corresponds to $L_{3 ; 5}^{\mathrm{I}}$. The other integrals possess lower-dimensional algebras. 


\section{Concluding Remarks}

In this work we have provided the algebraic structure of first integrals of the free particle or any scalar linearizable, via point transformation, ODE. Firstly, we derived the relationship between the symmetries and the first integrals of the free particle equation. By analyzing this classifying relation (3.6), we were able to establish the number of symmetries possessed by any first integral of the free particle equation. We obtained the important result that the symmetries admitted by a first integral can be $0,1,2$, or 3 . It was observed that the zero symmetry case was rather surprising or unexpected as one does not have a route to the integration of the equation due to the lack of any symmetry and this too for the simplest equation. The one- and two-symmetry cases were not unique; there were many first integrals with differing one- and two-symmetry structures. These were carefully discussed. Finally, we studied completely the situation when a first integral has three symmetries. We used the classification of realizations in the plane adapted as free particle symmetries. We showed that the only three-dimensional algebra admitted by a first integral of the free particle equation is $L_{3 ; 5}^{\mathrm{I}}$ which is admitted by the functionally independent integrals $I_{1}$ and $I_{2}$ as well as their quotient $I_{2} / I_{1}$. Although this triplet of symmetries was discovered before in the seminal work of Leach and Mahomed [4], these authors did not prove that it was unique nor the maximum algebra. However, they did emphasize the important result that the algebras of the triplets of symmetries were isomorphic and that the three triplets of symmetries generate the $\operatorname{sl}(3, R)$ symmetry of the equation. We showed that the maximum algebra is indeed the threedimensional algebra $L_{3 ; 5}^{\mathrm{I}}$ by completely analyzing all representations of the three-dimensional algebras.

\section{Acknowledgments}

K. S. Mohamed thanks the University of the Witwatersrand and the NRF of South Africa for financial support. E. Momoniat is grateful to the NRF for a research grant.

\section{References}

[1] S. Lie, "Klassifikation und Integration von gewöhnlichen Differentialgleichungen zwischen $x, y$, die eine Gruppe von Transformationen gestatten," Archiv Der Mathematik, vol. 8-9, p. 187, 1883.

[2] F. M. Mahomed, "Symmetry group classification of ordinary differential equations: survey of some results," Mathematical Methods in the Applied Sciences, vol. 30, no. 16, pp. 1995-2012, 2007.

[3] F. M. Mahomed and P. G. L. Leach, "Symmetry Lie algebras of $n$th order ordinary differential equations," Journal of Mathematical Analysis and Applications, vol. 151, no. 1, pp. 80-107, 1990.

[4] P. G. L. Leach and F. M. Mahomed, "Maximal subalgebra associated with a first integral of a system possessing sl(3,R) symmetry," Journal of Mathematical Physics, vol. 29, no. 8, pp. 1807-1813, 1988.

[5] K. S. Govinder and P. G. L. Leach, "The algebraic structure of the first integrals of third-order linear equations," Journal of Mathematical Analysis and Applications, vol. 193, no. 1, pp. 114-133, 1995.

[6] A. H. Kara and F. M. Mahomed, "A basis of conservation laws for partial differential equations," Journal of Nonlinear Mathematical Physics, vol. 9, supplement 2, pp. 60-72, 2002.

[7] A. M. Tresse, "Sur les invariants différentiels des groupes continus de transformations," Acta Mathematica, vol. 18, no. 1, pp. 1-88, 1894.

[8] F. M. Mahomed and P. G. L. Leach, "The Lie algebra $s l(3, R)$ and linearization," Quaestiones Mathematicae, vol. 12, no. 2, pp. 121-139, 1989.

[9] F. M. Mahomed and P. G. L. Leach, "The linear symmetries of a nonlinear differential equation," Quaestiones Mathematicae, vol. 8, no. 3, pp. 241-274, 1985. 
[10] F. M. Mahomed and P. G. L. Leach, "Lie algebras associated with scalar second-order ordinary differential equations," Journal of Mathematical Physics, vol. 30, no. 12, pp. 2770-2777, 1989.

[11] N. H. Ibragimov and F. M. Mahomed, "Ordinary differential equations," in CRC Handbook of Lie Group Analysis of Differential Equations, N. H. Ibragimov, Ed., vol. 3, p. 191, CRC Press, Boca Raton, Fla, USA, 1996.

[12] F. M. Mahomed, A. H. Kara, and P. G. L. Leach, "Lie and Noether counting theorems for onedimensional systems," Journal of Mathematical Analysis and Applications, vol. 178, no. 1, pp. 116-129, 1993. 


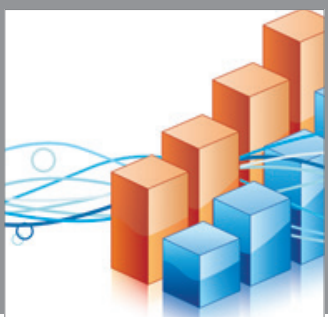

Advances in

Operations Research

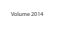

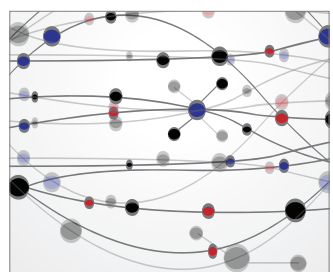

\section{The Scientific} World Journal
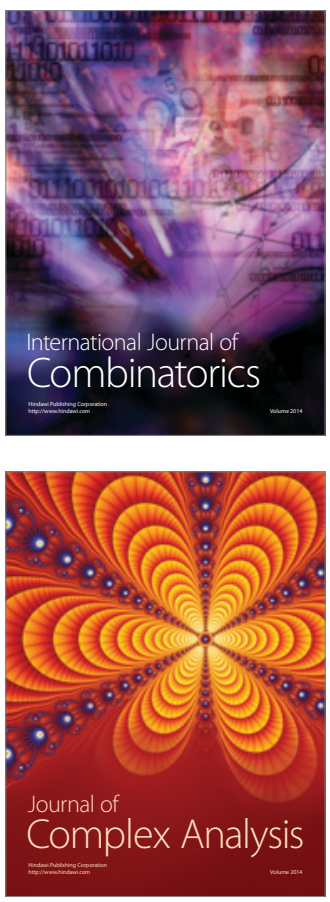

International Journal of

Mathematics and

Mathematical

Sciences
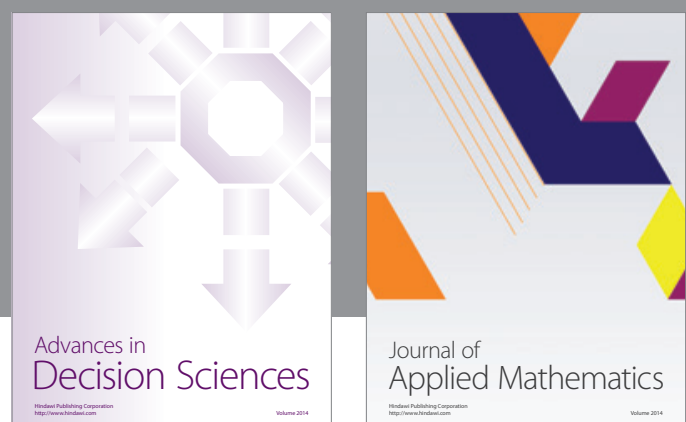

Journal of

Applied Mathematics
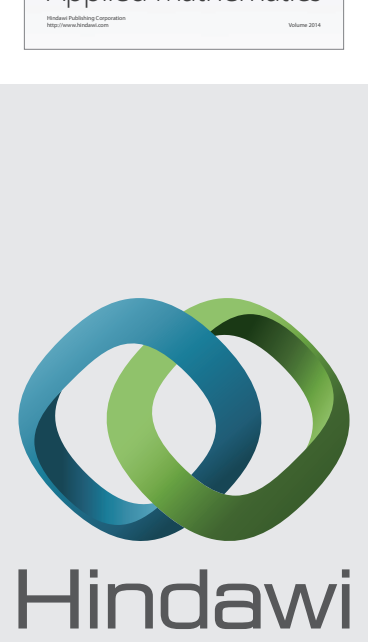

Submit your manuscripts at http://www.hindawi.com
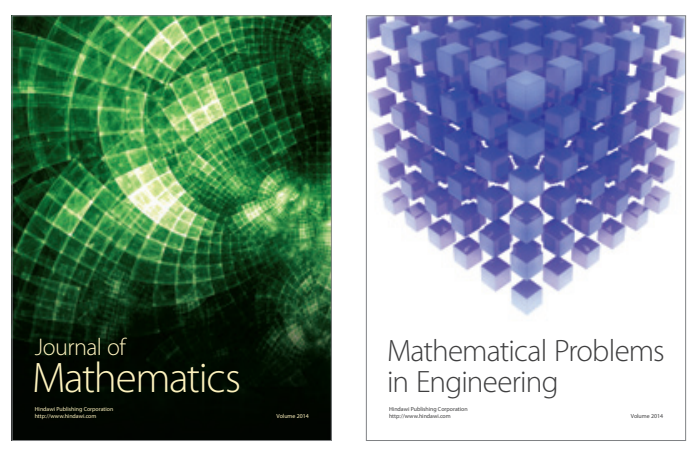

Mathematical Problems in Engineering
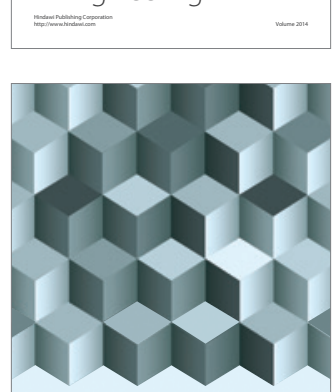

Journal of

Function Spaces
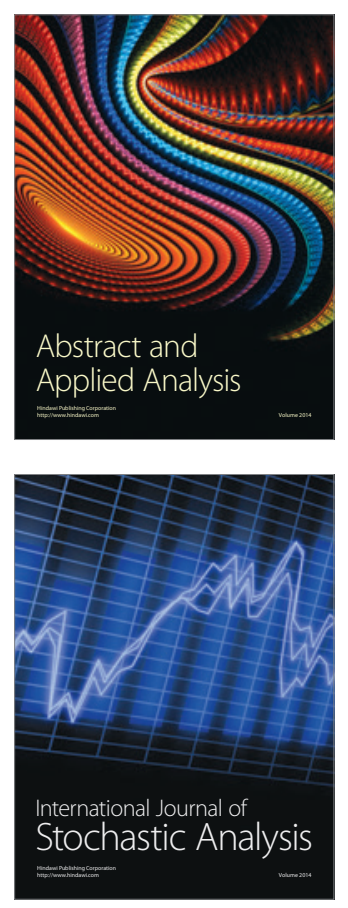

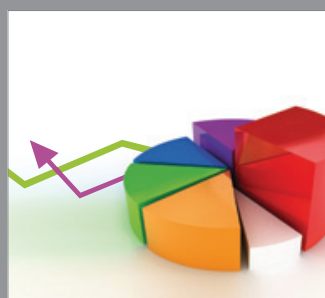

ournal of

Probability and Statistics

Promensencen
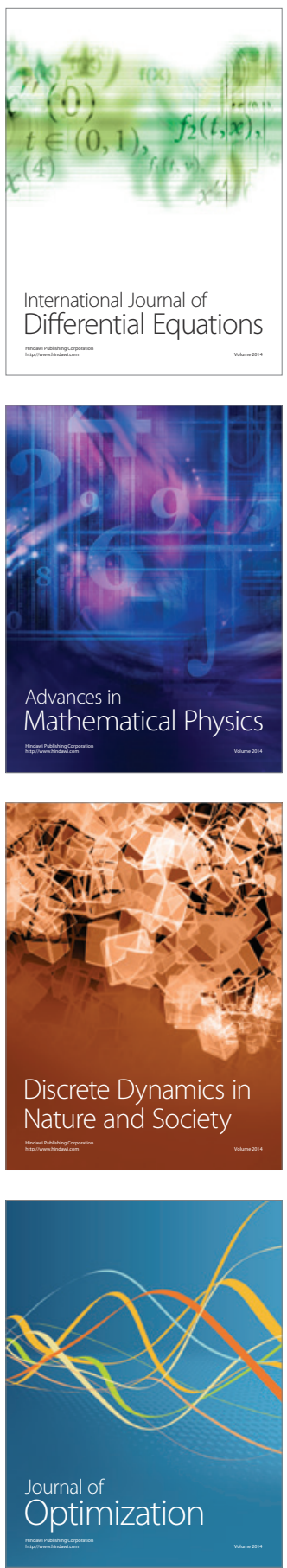